\title{
Effects of the recombinant allergen rDer $f$ on neuro-endocrino-immune network in asthmatic mice
}

\author{
YONG-QIAN JIANG, ZHI-XIANG ZHOU, YOU-LIN JI
}

Department of Respiratory, the First People's Hospital of Yancheng City, Jiangsu Yancheng, PR China

\begin{abstract}
Severe and life-threatening side effects can occur in patients receiving allergen-specific immunotherapy (SIT), and recombinant allergens made from cDNA have been used in clinical trials for ten years and appear promising for SIT. The aim of this study is to explore the effects of the recombinant allergen Der 2 (the group 2 allergen from Dermatophagoides farinae) on the neuro-endocrino-immune network in asthmatic mice. Twenty-eight mice were divided into four groups $-A, B, C$ and $D$. To induce asthma, a crude extract of $D$. farinae was injected intraperitoneally into the mice in groups $B$, $C$ and D. Later, the crude extract or recombinant allergen $r D e r f 2$ was given to groups $C$ and $D$, respectively. Normal saline was given to groups A and B. Serum corticotropin-releasing hormone (CRH), adrenocorticotropic hormone (ACTH), corticosterone (CORT), interleukin 4 (IL-4), and interferon $\gamma$ $(I F N-\gamma)$ were detected by immunoassay and the pathological change of lung tissue was observed by hematoxylin and eosin (HE) staining. Serum CRH, ACTH, CORT, and IFN- $\gamma$ were highest in healthy group A but lowest in asthma group B. Treatment with the crude extract or recombinant allergen $r D e r$ $f 2$ significantly attenuated this response in asthmatic mice, but there was no difference between the two treatments $(p>0.05)$. Serum IL-4 was elevated in asthma group B but lowest in healthy group A. Treatment with the crude extract or recombinant allergen $r D e r f 2$ significantly attenuated this response in asthmatic mice, but there was no significant difference between the two treatments $(p>0.05)$. However, lung pathology as measured histologically (Underwood Score) showed that rDer f 2-treatment was significantly better than crude extract treatment $(p<0.05)$. In brief, recombinant allergen Der $f 2$ can strengthen the function of hypothalamus-pituitary-adrenal (HPA) axis, affect the balance of Th1 and Th2 cytokines, and reduce pulmonary inflammation in asthmatic mice.
\end{abstract}

Key words: Dermatophagoides farinae, recombinant allergen, asthma, neuro-endocrino-immune network.

(Centr Eur J Immunol 2014; 39 (3): 294-298)

\section{Introduction}

Allergic asthma is an IgE-mediated disease caused by a type I hypersensitivity characterized by eosinophil accumulation in lung, excessive mucus secretion, and airway hyper-responsiveness. Its pathogenesis involves nervous, endocrine and immune systems [1]. Studies have shown that neuroendocrine hormones also regulate asthma and its pathogenesis. In asthma patients, glucocorticoids increase after early contact with allergens. However, patients with repeated asthma have dysfunction of the hypothalamus-hypophysis-adrenal gland (HPA) axis which impairs their ability to respond to stress. Secretion dysfunction of cortical hormones can aggravate asthma severity and then patients become more dependent on exogenous glucocorticoids; this vicious cycle can manifest in repeated asthma attacks.
Exogenous glucocorticoids are still the most effective drugs for asthma treatment. However, long-term systemic administration (repeated inhalation of high-dose glucocorticoids) can significantly inhibit the HPA axis of patients with asthma and is related to a decreased lung function [2, 3]. Therefore, it is important to discover a drug that can promote the secretion of endogenous glucocorticoids or have effects similar to corticosteroids. Secretion of glucocorticoids is mainly regulated by the HPA axis. Immune system stimulation increases cytokine expression such as proinflammatory cytokine interleukin 6 (IL-6). However, these cytokines can also activate the HPA axis to increase secretion of adrenocorticotropic hormone (ACTH) and corticosterone (CORT) to inhibit inflammation. Collectively, these mechanisms form the NEI network regulation of asthma [4]. 
With development and maturation of molecular biology techniques, studies about genetically-engineered allergens have become the mainstream in allergological studies. In the past 10 years, component genes of 19 kinds of Dermatophagoides ("dust mite") allergens have been cloned using reporter systems in Escherichia coli, yeast, and baculovirus. It has also been proven that allergens prepared by genetic engineering technology have similar biologic activity to natural allergens [5]. In this study, recombinant allergens were administered to mice with asthma via intraperitoneal injection to observe the effects on the NEI network (mainly the HPA axis and relevant cytokines) and lung pathology.

\section{Material and methods}

\section{Experimental animals, reagents and apparatus}

Female C57BL/6 mice (aged 5 to 6 weeks, body weight $20.6 \pm 2.3 \mathrm{~g}$ ) were purchased from the Shanghai Animal Center of the Chinese Academy of Sciences. Animals were housed under sterile conditions in the Laboratory Animal Center of the Shanghai Second Military Medical University. The crude extracts of Dermatophagoides farinae and prepared recombinant protein of the group 2 allergen from D. farinae (Der f 2) was provided by Dr. Yubao Cui [6]. Mouse corticotropin-releasing hormone (CRH) and adrenocorticotropic hormone (ACTH) radioimmunoassay kits were produced by DSL (USA). Mouse corticosterone (CORT), interleukin 4 (IL-4), interferon- $\gamma$ (IFN- $\gamma$ ) ELISA kits were produced by R\&D Systems (USA). Results were read with an ELx800 microplate reader from Bio-Tek (USA).

\section{Methods}

\section{Animal groups and treatments}

C57BL/6 mice $(n=28)$ were divided into four groups by the digit randomization method, $n=7$ in each group: normal healthy control group (A); asthma control group (B); crude extract-treated asthma group (C); and rDer f 2treated asthma group (D). To induce asthma in groups B, $\mathrm{C}$, and $\mathrm{D}$, animals were given a crude extract of $D$. farinae $(50 \mu \mathrm{l})$ on day 1 and day 17 via intraperitoneal injection [6]. On day $15,16,17$, and 18 , the mice in groups B, C, and $\mathrm{D}$ were anesthetized with $0.1 \mathrm{ml} 0.5 \%$ pentobarbital sodium via intraperitoneal injection, and then stimulated with a crude extract of $D$. farinae [6] $50 \mu \mathrm{l} /$ per injection/ day. From day 31 to day 33, animals in group C and D were give a crude extract of $D$. farinae and rDer $\mathrm{f} 2$ via intraperitoneal injection while animals in group A and B were given saline. On day 39 and 46, all asthma model animals (groups B, C, and D) were given a crude extract of D. farinae via intranasal drip for stimulation while animals in group A were given saline by intranasal drip.

\section{Detection of indicators in serum}

Twenty-four hours after the last intranasal drip, $0.1 \mathrm{ml}$ of $0.5 \%$ pentobarbital sodium was given via intraperitoneal injection. Eyeballs of the mice were removed after being anesthetized and blood was taken. The serum was separated, aliquoted, and stored at $-20^{\circ} \mathrm{C}$ Radioimmunoassay method was used to detect serum $\mathrm{CRH}$ and $\mathrm{ACTH}$ and ELISA method was used to detect serum CORT, IL-4, and IFN- $\gamma$. The specific steps were performed according to the kit instructions.

\section{Pathological detection of lung tissue}

The bronchus and lung tissue of the mice were cut, fixed in $10 \%$ neutral formalin solution for $\geq 24$ hours, then dehydrated, embedded, and prepared into conventional paraffin sections. Sections were stained with hematoxylin and eosin (HE) stain and sealed with neutral gum. Pathological changes of lung tissue were observed by microscope at $100 \times$ power.

\section{Statistical analysis}

All the test results were analyzed with SPSS 13.0 statistical software package and expressed as mean \pm standard deviation $(\bar{x} \pm s)$. Single factor variance analysis was applied to examine serum CRH, ACTH, CORT, IL-4, IFN- $\gamma$ values and histology-determined Underwood Standard Score of lung pathology. Pair-wise comparisons were conducted with the Student-Newman-Keuls test. The above hypothesis test was two-sided; the test level $\alpha$ was 0.05 with $p<0.05$ considered statistically significant.

\section{Results}

\section{Serum values}

Tables 1 and 2 show that the difference in serum CRH, ACTH, CORT, IL-4, IFN- $\gamma$ of mice among groups was significantly different $(p<0.05)$. Pair-wise comparison showed that serum CRH, ACTH, CORT, and IFN- $\gamma$ in groups $\mathrm{C}$ and $\mathrm{D}$ were significantly increased $(p<0.05)$ compared to group B, but still lower than that of group A $(p<0.05)$, with no statistically significant difference between groups C and D $(p>0.05)$. Compared to group B, serum IL-4 in groups $\mathrm{C}$ and D was significantly decreased $(p<0.05)$ but still higher than group $\mathrm{A}(p<0.05)$, with no statistically significant difference between groups $\mathrm{C}$ and $\mathrm{D}(p>0.05)$.

\section{The pathological changes in lung tissue}

In asthma group B, results showed thickened capillaries in the alveolar wall and the alveolar septum with an increased number of eosinophilic granulocytes, leukomonocytes and other inflammatory cells nearby bronchioles and blood vessels. Inflammatory cells were reduced in groups 
Table 1. Serum CRH, ACTH, CORT levels among groups $(\bar{x} \pm \mathrm{s})$

\begin{tabular}{ccccc}
\hline Group & $\boldsymbol{n}$ & $\mathbf{C R H}(\boldsymbol{\mu g} / \mathbf{l})$ & ACTH $(\mathbf{n g} / \mathbf{l})$ & CORT $(\boldsymbol{\mu g} / \mathbf{m l})$ \\
\hline $\mathrm{A}$ & 7 & $3.47 \pm 0.40$ & $127.45 \pm 18.67$ & $159.68 \pm 16.14$ \\
\hline $\mathrm{B}$ & 7 & $2.18 \pm 0.31^{*}$ & $78.38 \pm 18.19^{*}$ & $103.48 \pm 9.30^{*}$ \\
\hline $\mathrm{C}$ & 7 & $2.77 \pm 0.55^{* \#}$ & $103.88 \pm 20.82^{* *}$ & $131.16 \pm 27.81^{* *}$ \\
\hline $\mathrm{D}$ & 7 & $2.85 \pm 0.30^{* \#}$ & $101.45 \pm 12.42^{* \#}$ & $133.28 \pm 23.94^{* *}$ \\
\hline$F$ & \multicolumn{5}{c}{0.927} & 8.889 & 8.720 \\
\hline$p$ & 0.001 & 0.001 & 0.001 \\
\hline${ }^{*} p<0.05, v s$. A group * $^{*} p<0.05, v s$. B group
\end{tabular}
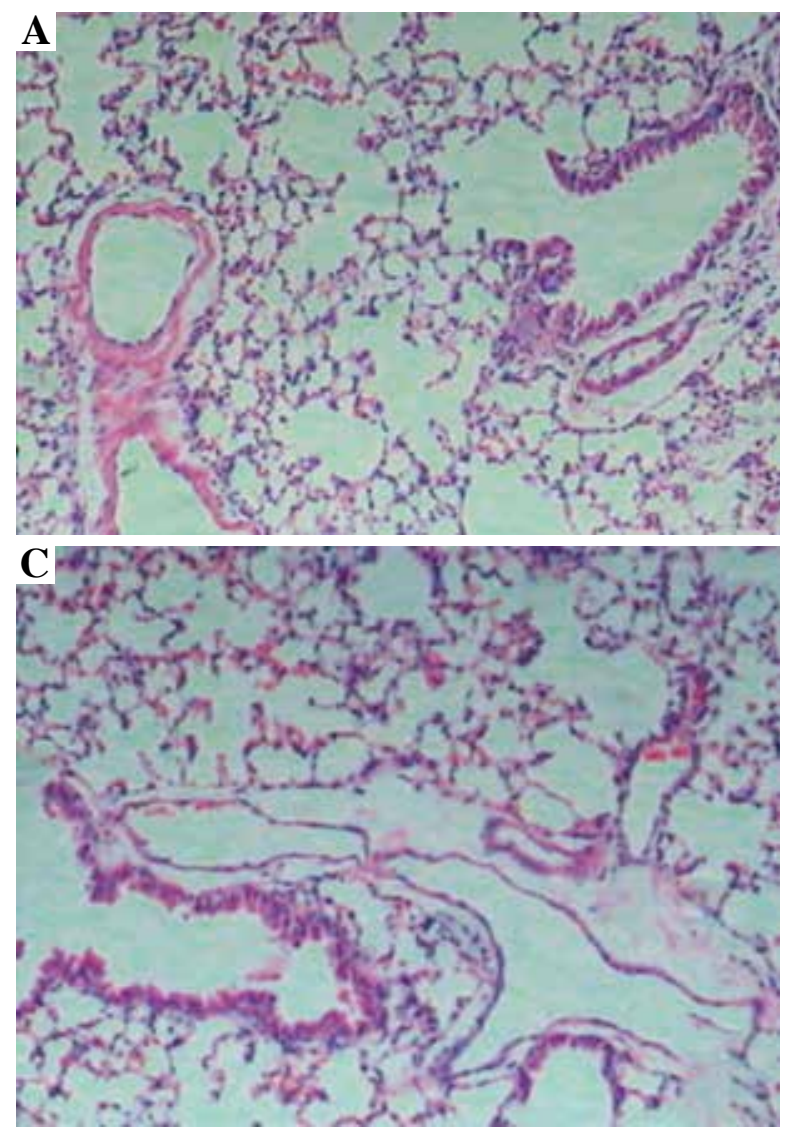

Fig. 1. Pathological sections of lung tissues of mice (H\&E staining, 100`). Note: A) normal control group; B) model control group; C) crude extract treatment group; D) rDer f 2 treatment group

C and D. Group C had more significant changes, and the severity of inflammatory cell infiltration in bronchi, bronchioles and accompanying the vessels decreased, eosinophilic granulocytes reduced, and the edema improved, seen in Fig. 1. The Underwood Standard Scores of lung tissue in group A, B, C, and D were $0.17 \pm 0.01,3.31 \pm 0.10,1.35$ \pm 0.18 , and $1.83 \pm 0.08$, respectively, with significant differences across groups ( $p=0.001, F=958.328$ ). The pairwise comparison showed that the Underwood Standard Scores of lung tissue in groups $\mathrm{C}$ and $\mathrm{D}$ were significantly de-
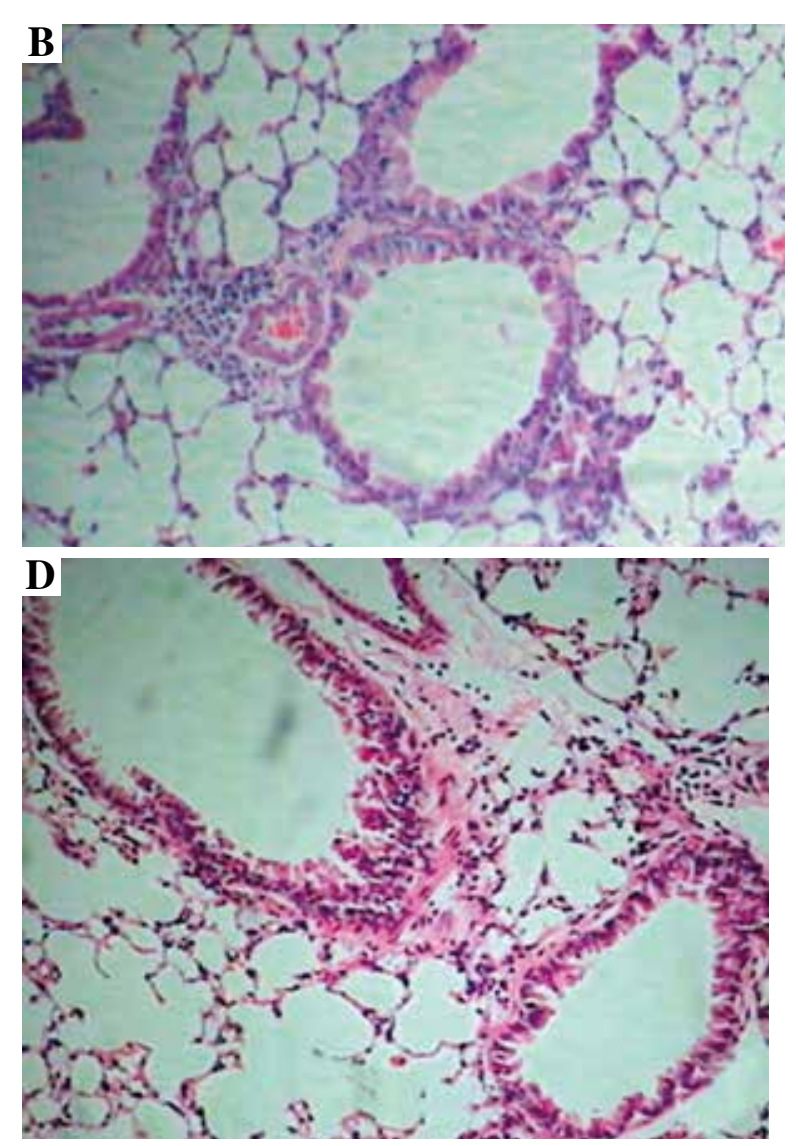

Table 2. Serum IL-4 and FN- $\gamma$ levels among groups $(\bar{x} \pm \mathrm{s})$

\begin{tabular}{cccc}
\hline Group & $\boldsymbol{n}$ & $\mathbf{I L - 4}(\mathbf{p g} / \mathbf{m l})$ & IFN- $\gamma(\mathbf{p g} / \mathbf{m l})$ \\
\hline A & 7 & $30.37 \pm 3.84$ & $23.24 \pm 2.61$ \\
\hline B & 7 & $46.69 \pm 4.89^{*}$ & $9.86 \pm 1.95^{*}$ \\
\hline C & 7 & $38.05 \pm 3.36^{* \#}$ & $14.39 \pm 3.91^{* \#}$ \\
\hline D & 7 & $36.91 \pm 3.69^{* \#}$ & $13.49 \pm 1.58^{* \#}$ \\
\hline$F$ & & 19.795 & 31.796 \\
\hline$p$ & & 0.001 & 0.001 \\
\hline
\end{tabular}

${ }_{p}<0.05$, vs. A group; $p<0.05$, vs. B group

creased $(p<0.05)$ compared to asthma control group B, but still higher than healthy group A $(p<0.05)$. Finally, the scores of groups $\mathrm{C}$ and $\mathrm{D}$ were significantly different $(p<0.05)$.

\section{Discussion}

Asthma involves chronic inflammation of the airway characterized by airway hyper-responsiveness and airflow obstruction. Asthma has a complex pathogenesis that in- 
volves a variety of inflammatory cells and factors [7, 8]. At present, the mechanisms of airway inflammation include theories of immunogenic and neurogenic inflammation [9]. Neuropeptide transmitters released by airway sensory nerve endings and cytokines can cause contraction of airway smooth muscle and gland endocrine activation, further promoting airway hyper-responsiveness. Current research suggests that asthma pathogenesis includes local and systemic inflammation but also involves the central nervous system to constitute a neuroendocrine-immune regulatory network [10].

This study used a crude extract of $D$. farinae to prepare a mouse model of asthma. Results showed that serum $\mathrm{CRH}, \mathrm{ACTH}$, and CORT levels in the asthmatic group B were significantly lower than those of the healthy mice in group A. Results from this study were consistent with those obtained in a rat model of asthma by Jeppop et al. [11]; this further affirms neuroendocrine involvement in asthma. Mice given a crude extract of $D$. farinae and $\mathrm{rDer} f 2$ (groups $\mathrm{C}$ and $\mathrm{D}$, respectively) showed increased serum $\mathrm{CRH}, \mathrm{ACTH}$, and CORT, though the levels did not reach those in the healthy control group.

Interleukin 4 is mainly secreted by Th2 cells, which primarily mediate humoral immunity and play a leading role in allergic reactions. Interleukin 4 promotes antigen-specific B cells to secrete IgE antibodies and has an important effect on asthma incidence [12]. Interferon $\gamma$ is mainly secreted by Th1 cells and can inhibit Th2 cell differentiation; this effect could inhibit the biological effects of IL-4 and other cytokines that act through IFN- $\gamma$ to inhibit IgE production and an asthmatic reaction. Interferon $\gamma$ is a very important inhibitory molecule in the immune regulation of asthma [13]. Meanwhile, IFN- $\gamma$ has ACTHlike effects in that it can stimulate the adrenal cortex to increase plasma corticosterone [14].

In group B mice with asthma, serum Th2-type cytokine IL-4 was significantly increased compared to healthy mice in group A, while Th1-type cytokine IFN- $\gamma$ was significantly decreased, suggesting that IL- 4 and IFN- $\gamma$ play an important role in asthma development. In this study, serum IL-4 of mice treated with a crude extract of $D$. farinae and rDer $\mathrm{f} 2$ (groups $\mathrm{C}$ and D) was decreased while serum IFN- $\gamma$ was significantly increased. These results suggest that rDer $\mathrm{f} 2$ had similar effects to a crude extract of $D$. $f a$ rinae - inhibited Th2 response and enhanced Th1 response - to reduce asthma pathogenesis.

Chronic airway inflammation in asthma involves a variety of cells, and its histopathological changes involve the airways, pulmonary interstitial vessels and pulmonary alveoli [15]. In this study, the crude extract was given to the sensitized mice via intraperitoneal injection, and inflammatory cell infiltration was observed around the bronchioles and small blood vessels of mice in group B with increased intraluminal exudate and significant edema. These observations confirmed that the asthma animal model was successfully established. No inflammatory cells were observed in group A. Inflammatory cells in groups C and D were reduced, eosinophilic granulocytes were reduced, and the severity of inflammatory cell infiltration in bronchi, bronchioles and accompanying the vessels was decreased. These results suggest that both the crude extract of $D$. $f a$ rinae and $\mathrm{rDer} f \mathrm{can}$ reduce lung inflammation. The effects of rDer $\mathrm{f} 2$ do not match those of the crude extract of $D$. farinae. This result is likely because the crude extract was used to sensitize the mice and prepare the asthma model, and that this crude extract has many other components besides Der $\mathrm{f} 2$. The roles of these other components of $D$. farina for sensitization against asthma require further studies.

In summary, prepared rDer $\mathrm{f} 2$ can enhance the function of HPA axis and regulate the balance between Th1 and Th2 cytokines. The pathological observations from lung tissues confirm that these mechanisms correlate with reduced allergic systemic inflammation and lung pathology.

The authors declare no conflict of interest.

\section{References}

1. Derv RE, Ulanova M, Puttagunta L, et al. (2004): Frontline: Inhibition of allergen-induced pulmonary inflammation by the tripeptide feG: a mimetic of a neuro-endocrine pathway. Eur J Immunol 34: 3315-3325.

2. Casale TB, Nelson HS, Stricker WE, et al. (2001): Suppression of hypothalamic-pituitary-adrenal axis activity with inhaled flu-nisolide and fluticasone propionate in adult asthma patients. Ann Allergy Asthma Immunol 87: 379-385.

3. Lange P, Scharling H, Ulrik CS, Vestbo J (2006): Inhaled corticosteroids and decline of lung function in community residents with asthma. Thorax 61: 100-104.

4. Zhao FD, Dong JC, Xie JY (2007): Effects of Chinese Herbs for Replenishing Shen and Strengthening Qi on Some Indexes of Neuro-endocrino-im-mune Network in Asthmatic Rat. Zhongguo Zhong Xi Yi Jie He Za Zhi 27: 715-719 (In Chinese).

5. Thomas WR, Smith WA, Hales BJ, et al. (2002): Characterization and Immunology of house dust mite allergens. Int Arch Allergy Immunol 129: 1-18.

6. Yu-bao C, Zhou Y, Weihong S, et al. (2010): Cloning, expression, and analysis of the group 2 allergen from Dermatophagoides farinae from China. An Acad Bras Cienc 82: 941-951.

7. Holgate ST. (2008): Pathogenesis of asthma. Clin Exp Allergy $38: 872-897$.

8. Finiasz M, Otero C, Bezrodnik L, Fink S (2011): The role of cytokines in atopic asthma. Curr Med Chem 18: 1476-1487.

9. Barnes PJ (2001): Neurogenic inflammation in the airways. Respir Physiol 125: 145-154.

10. Marshall GD (2004): Neuroendocrine mechanisms of immune dysregulation: application to allergy and asthma. Ann Allergy Asthma Immunol 93: S11-17.

11. Jessop DS, Renshaw D, Larsen PJ, et al. (2000): Substance P is involved in terminating the hypothalamo-pituitary-adrenal axis response to acute stress through centrally located neurokinin-1 receptors. Stress 3: 209-220. 
12. Ngoc PL, Gold DR, Tzianabos AO, et al. (2005): Cytokines, allergy, and asthma. Curr Opin Allergy Clin Immunol 5: 161166.

13. Kumar RK, Webb DC, Herbert C, Foster PS (2006): Interferon-gamma as a possible target in chronic asthma. Inflamm Allergy Drug Targets 5: 253-256.

14. Koch M, Witzenrath M, Reuter C, et al. (2006): Role of local pulmonary IFN-gamma expression in murine allergic airway inflammation. Am J Respir Cell Mol Biol 35: 211-219.

15. Xu J (2007): New advances in the pathogenesis of asthma. Zhonghua Jie He He Hu Xi Za Zhi 30: 490-493 (In Chinese). 